\title{
A Genetic Algorithm Based Intuitionistic Fuzzification Technique for Attribute Selection
}

\author{
S. Senthamilarasu' ${ }^{1}$ and M. Hemalatha ${ }^{2^{*}}$ \\ 1,2 Department of Computer Science, Karpagam University, Coimbatore-21, TN, India; \\ s.a6asu@gmail.com', csresearchhema@gmail.com²
}

\begin{abstract}
This paper initiates perceptions and algorithms of feature selection, survey of existing feature selection algorithms and assesses diverse algorithms with a classifying frame based on search approaches, valuation criteria, and provides strategy in selecting feature selection algorithms. A unifying platform is projected to continue our efforts headed for building an incorporated system for intelligent feature selection. Feature selection intends to reduce the dimensionality of patterns for classification by choosing the most informative instead of irrelevant and/or redundant features. In this proposed work Intuitionistic fuzzy based feature clustering is proposed for grouping features based on the degree of membership and degree of indeterminacy among the attributes and clusters. In this proposed work a novel approach which uses an Intuitionistic fuzzy version of k-means has been introduced for grouping interdependent features. Genetic algorithm reinstating which is the variation of traditional genetic algorithm is then applied to appraise whether the measured feature is independent of class labels; hence, it leads to eliminate unrelated clusters to classification process and progress the selection of features. The proposed method achieves improvement on classification accuracy and perhaps to select less number of features which show the way to simplification of learning task to a big extent. The Experiment results have been demonstrated by the good performance and also find good enough subset features of this method on using UCI benchmark datasets that are for data mining methods such as Breast Cancer, Sensor and Iris Records.
\end{abstract}

Keywords: Feature Selection, Cluster, Genetic, K-means, Fuzzy, Intuitionistic.

\section{Introduction}

Renovation of information may fluctuate the organization grows. The fluctuation of data stream $[1,2,3]$ might correctly affect the statistics, no problem. Otherwise, it causes the organization. The transformation of data during the network, at that time, the insertion or updating of data is very important i.e. Concept drift. Concept drifting of each datum may cause the accuracy of the classifier and it's also difficult to analyze and monitor the data. Because of, data may loss while input rates higher than the memory capacity. The dimensionality reduction [4] i.e. Feature Selection (FS) [5] is the way to reduce the input rate and to bring up with the compact of memory capacity. FS is one of the preprocessing and post processing essential steps in the Data Mining Techniques. The both processing steps are used to increase the classifier accuracy. Here, FS performs priory step for reducing dimensionality for deducting the higher input rates.

The purpose of the FS is to find out (or) choose the best subset feature from all other features in the dataset. Selecting possible features are discovering more information in the large data base. The main aim of the FS is to detect and remove the noise, irrelevant features and increase the accuracy building model. For large data sets, we use filters to select the best features score based on a rule that measures each feature independently. FS is a key step for many machine learning algorithms, especially when the

\footnotetext{
* Corresponding author:
}

M. Hemalatha (csresearchhema@gmail.com) 
data are high-dimensional [6]. Choosing a small subset of features i.e. adequate to describe the target according to the way the computing feature selection broadly classified into three categories as Filter, wrapper and embedded approach [7]. FS method typically consists of two basics [8] [9].

- Evaluation criterion, to evaluate a measure to choose best subset feature $\mathrm{S}$.

- Search strategy, a procedure to produce candidate subset $\mathrm{S}$ for searching the feature power set.

Many features might be irrelevant and possibly is not favorable to classification. Also, redundancy among the features is common $[10,11]$. The presence of irrelevant and redundant features not only slows down the learning algorithm but also confuses it by causing it to over fit the training data. In other words, ignoring (or) removing irrelevant and redundant features make the classifier's design simple, improves its prediction performance and its computational efficiency [12].

\section{Feature Selection}

\subsection{Feature Selection Objectives}

- Different feature selection algorithms may have various objectives to achieve. The following is a list of common objectives used by researchers.

- Identify the minimally sized feature subset that is essential and adequate to the target concept.

- From the Selected subset of $S$ features from a set of $N$ features, $S<\mathrm{N}$, such that the value of a criterion function is optimized over all subsets of size $S$.

- Choose a subset of features for improving prediction accuracy or decreasing the size of the structure without significantly decreasing the prediction accuracy of the classifier built using only the selected features

- Select a small subset such that the resulting class distribution, given only the values for the selected features, is as close as possible to the original class distribution given all feature values.

This Section describes the feature Selection problem and presents various methods based on the literature review for its solution. E.g. Filter, Wrapper and Embedded approaches [7] [13]. The main focus of the FS is selecting the subsets of features which are used to construct the models $[14,15,16,17]$. FS basically evaluates one in a thrice, which is defined earlier. In Filter approach measures independently, computes the attribute weights eventually, select the subset of features which have a high dependency on the target class and will have less correlation among them. FS is to perform using statistical technique over the data, accuracy of future, induced classifier. Therefore this approach filters out the bad (or) irrelevant features before inducting process. In wrapper approach generally based on the optimization of the accuracy rate, estimated by one of the following methods, hold-out, cross validation (or) Bootstrap and is to evaluate the goodness of subset of features being selected and it deploys inductive classification algorithms. a).It searches the space of all possible features. b) Accuracy of Selected subset of features. Its aim to find minimum discriminative features to reach high classification accuracy. In Embedded approach, a search optimal subset of the feature is to build into the classifier model construction, can be seen as a search in the combined space of feature subset and hypotheses and include interaction (or) work together with the classifier model. The performance of high-dimensional binary data based on the class dependent density based on feature ranking (FR) algorithm for feature elimination of binary data set [18]. The significance of the unsupervised FS is to motivate by the ensemble by clustering techniques, which combining the multiple clustering solutions for higher accuracy. Unsupervised FR method is solution for true clustering number when combining particular cluster ensemble algorithm and the knowledge of the true cluster number [19]. The importance of reducing the dimensionality to select the best subset by minimizing the error rate by Bayes method based on the non-parametric weights [20]. FS focused on labeled data based on the two level criterion i.e. Distance measure and dependency measure. After that, arrange the attributes, evolved with the constructed decision tree and generated small tree with acceptable accuracy [21]. FS is performed based on genetic search, is to work with the global search algorithm to increase the accuracy of the classifier model [22]. Hybrid approach i.e. Composed of two conventional algorithms are used to perform the FS. Genetic Algorithm (GA) [23, 24] and Support vector Machine (SVM) are working together effectively on the wrapper model. GA finds the best subset by applying evolutionary process and SVM deduced the data set. Hybrid approach performed by using dependency measure between attributes. By the process, fitness measures are replaced, where weak attribute is placed? and it increase the fitness of population [16, 24].Another way of performing GA using hybrid approach is to select the best subset features from the original dataset by feature pool method. 
A feature pool is a collection of features selected by genetic algorithm to select an optimal feature from the data set. Selected features are evaluated by multiple feature algorithms to form pool [25]. By performing online selection is not adapted for real world application because of accessing the data instances are high dimensional and too expensive to acquire the dataset. To solve the problem with deletion of Small or insufficient in quantity or amount of data which is not suitable for feature selection [26]. The major problem encountered in feature Selection, all possible features combination should be searched. This search space is exponential prohibitive for exhaustive search even with a moderate attribute. In this case the high computational cost, scalability because of the communication technology has been growth. So, transmitting data ten times bigger than past decades, time consuming, minimizing the redundancy among these features. These problems are still not solved in recent years. The background of the study focus on feature selection based on attributes and objectives are shown below [27] and also we follows the materials and methods are described about reducing the dimensionality reduction in various clustering methods such as K-means, Fuzzy K-means and Genetic algorithm.

\subsubsection{K-Means}

K-Means $[28,29]$ is one of the classical methods for unsupervised evolutionary DM algorithm that solves the well-known clustering problem in a simple way and it's commonly used in medical imaging, biometrics and other emerging fields. It automatically adapts the best understood clustering methods for observing the grouping objects [30]. The K-Means algorithm to form a group a collection of vectors into $\mathrm{K}$ groups and find a cluster center in each group. Finally we got constant cluster center in each group in an iterative way that a cost function shown in (1) is minimized.

$$
I=\sum_{r=1}^{k} J_{r}=\sum_{r=1}^{k}\left(\sum_{x \in G_{r}} d\left(x, C_{r}\right)^{2}\right)
$$

where, $k$ is the number of clusters, $G_{r}$ and $C_{r}$ are the set of $\mathrm{r}^{\text {th }}$ cluster and its associated center respectively. $X$ presents a data point and $d(a, b)$ is the distance functional (usually Euclidean distance) between $a$ and $b$.

In this method the value of $\mathrm{k}$ should be determined first. This value is fixed during the execution of the algorithm. The algorithm is summarized in the following steps:

Step1: Initializes the $\mathrm{k}$ cluster centers randomly
Step2: while the stop condition is not satisfied do the following:

a) Assign each vector to a cluster with nearest center.

b) Update cluster centers to the average of vectors belong to that cluster in order to decrease the cost function (2).

$$
\operatorname{Min}_{c_{k}} \sum_{i=1}^{N}\left\|x_{i}^{k}-C_{k}\right\|^{2}
$$

\subsubsection{Fuzzy K-Means}

Fuzzy K-Means (also called Fuzzy C-Means, Hard or Crisp Cluster) is a simplified extension of K-Mean Clustering algorithm $[31,32,33]$. Identifying the pattern structure and attempt to capture \& quantifying the non-random imprecision are performed in this algorithm [34].

$$
l(K, m)=\sum_{k=1}^{K} \sum_{i=1}^{N}\left(U_{k_{i}}\right)^{m} d^{\mathbf{2}}\left(x_{i}, c_{k}\right)
$$

where $\mathrm{K}$ is the number of Cluster, $\mathrm{m}$ is the parameter, $U_{k_{i}}$ Is the membership degree of $x_{i}$, in Cluster $\mathrm{K}, d^{2}\left(x_{i}, c_{k}\right)$ is the distance from $x_{i}$ controls $c_{k}$. The parameter in this equation are the centroid vector $c_{k}$ and the components of the membership vector $U_{k_{i}}$. Evaluate $U_{k_{i}}$ shows the belonging ratio to a cluster $\mathrm{K}$ and centroid $c_{k}$ belonging expression cluster $\mathrm{k}$.

In this method a data point can belong to several groups with a membership degree between 0 and 1 which is shown by a matrix $U_{k \times m}$ where $k$ is number of clusters and $m$ is number of data points such that the condition in (4) is satisfied.

$$
\sum_{r=1}^{c} U_{r i}=1, \forall i=1, \ldots, n
$$

where, $U_{r i}$ is the membership degree of $i^{\text {th }}$ pattern in $r^{\text {th }}$ cluster. The cost function used in this method is shown in the equation (5)

$$
l\left(U, c_{1}, \ldots, c_{k}\right)=\sum_{r=1}^{k} J_{r}=\sum_{r=1}^{k} \sum_{i}^{n} U_{r i}^{m} d\left(x_{i}, c_{r}\right)
$$

In order to minimize the cost function, membership degrees and cluster centers, in each iteration, are computed and the equations are (6) and (7)

$$
\begin{aligned}
U_{1} r i= & 1 /\left(\Sigma _ { 1 } ( l - 1 ) ^ { \dagger } k \overline { \overline { \mu } } \left(\left(d\left(x_{1} i, c_{1} r\right)\right) /\right.\right. \\
& \left.\left(d\left(x_{1} i, c_{1} l\right)\right)^{\dagger}(2 /((m-1)))\right)
\end{aligned}
$$




$$
C_{r}=\frac{\sum_{i-\mathbf{1}}^{k} u_{r i}^{m} x_{i}}{\sum_{j-1}^{k} u_{r j}^{m}}
$$

where, $x_{i}$ represents the $i^{\text {th }}$ pattern in dataset. Like K-Means, Fuzzy K-Means [35, 36] works on those objects which can be represented in $\mathrm{n}$-dimensional vector space and a distance measure is defined. This method performs the following steps.

Step1: Initializes the $\mathrm{k}$ fuzzy cluster centers randomly

Step2: while the stop condition is not satisfied do the following:

a) Compute the membership matrix $U$ according to the equation (6)

b) Update fuzzy cluster centers according to the equation (7)

\subsubsection{Genetic Algorithm}

Genetic Algorithm (GA) is based on the random sampling techniques to find the sub-optimal solutions. GA have been widely used in high dimensionality problem such as micro array data. GA operators namely Selection, Mutation and Crossover based on new offering which is initiated by initial chromosomes as parents. GA generates the sample population with certain number of subset of features, is randomly selected from the data set. The 'goodness' of each subset of features is evaluated in terms of the fitness, which is estimated by a classification algorithm. The certain number of subset of features that meet the fitness criterion can be found using an iterative scheme. For each iteration, the fitness of each subset of feature is computed. The subset of feature is chosen for crossover and mutation based higher probability, to select the subset of features with better fitness, and versa. A new offspring (subset of features) is generated by making the crossover between two selected subset of features. After a crossover is performed, mutation is employed. Mutation changes randomly the new offspring in order to prevent falling all solutions in population into a local optimum. If the fitness of the subset of feature is satisfied, one near optimal subset of features is obtained, and a new population is generated. The computation is terminated until the predefined $\mathrm{K}$ near optimal subset of features is found.

\subsection{Feature Selection Procedure}

A typical feature selection procedure consists of four basic steps:

1) Subset generation

2) Subset evaluation
3) Stopping criterion and

4) Result validation.

The process begins with subset generation that employs a certain search strategy to produce candidate feature subsets. Then each candidate subset is evaluated according to a certain evaluation criterion and compared with the previous best one. If it is better, then it replaces the previous best. The process of subset generation and evaluation is repeated until a given stopping criterion is satisfied. Finally the selected best feature subset is validated by prior knowledge or some test data. Search strategy and evaluation criterion are two key topics in the study of feature selection.

To cluster features, the k-modes algorithm is utilized which is similar to the well known clustering method, $\mathrm{k}$-means $[37,38]$. Mode of each cluster is defined as one of its features which have the largest multiple interdependence redundancy measure among other features in that cluster. The multiple inter-dependent redundancy measure is calculated for each feature by the following equation (8)

$$
\operatorname{MR}\left(A_{i}\right)=\sum_{\left.A_{j} \in \text { cluster( } i\right)} R\left(A_{i}: A_{j}\right)
$$

where, Cluster (i) is the set of features which are in the same cluster with $\mathrm{Ai}$ and is the interdependence measure between the two features, $A_{i}$ and $A_{j}$, which is defined by the equation (9)

$$
R\left(A_{i}: A_{j}\right)=\frac{I\left(A_{i}: A_{j}\right)}{H\left(A_{i}: A_{j}\right)}
$$

where, $I\left(A_{i}: A_{j}\right)$ is the mutual information between $A_{i}$ and $A_{j}$ as computed in the equation (10)

$$
\begin{aligned}
& I\left(A_{1} i: A_{1} j\right)=\Sigma_{\downarrow}(k-1)^{\dagger}\left(m_{1} i\right)=\sum_{\downarrow}(l-1)^{\dagger}\left(m_{1} j\right) \\
& \text { 【 } \operatorname{Pr}\left(A_{\downarrow} i=v_{\downarrow} i k{ }^{\top} A_{\downarrow} j=v_{\downarrow} j l\right) \rrbracket \\
& \log \frac{\operatorname{Br}\left(A_{i}=v_{i k}\left(A_{j}=v_{j l}\right)\right)}{\operatorname{Pr}\left(A_{i}=v_{i k}\right) \operatorname{Pr}\left(A_{j}=v_{j l}\right)}
\end{aligned}
$$

$H\left(A_{i}: A_{j}\right)$ is joint entropy of $A_{i}$ and $A_{j}$ which is given by the equation (11)

$$
\begin{aligned}
& H\left(A_{1} i: A_{1} j\right)=\Sigma_{1}(k-1)^{\top}\left(m_{1} i\right)=\Sigma_{l}(l-1)^{\boldsymbol{\top}}\left(m_{1} j\right)
\end{aligned}
$$

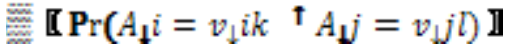

$$
\begin{aligned}
& \log \operatorname{Pr} \llbracket A_{i}=v_{i k}\left(A_{j}=v_{j l}\right)
\end{aligned}
$$

$H\left(A_{i}: A_{j}\right)$ is used to normalize $I\left(A_{i}: A_{j}\right)$ in (10). The larger value for $I\left(A_{i}: A_{j}\right)$, the higher interdependency of two features, $A_{i}$ and $A_{j}$. Hence, there should be some pairs of values 
for these features which are simultaneously visited with high frequency and other pairs are less probable. Therefore, having one of the values, other one may be approximated considering value pairs with high probability.

\section{The Proposed Intuitionistic Fuzzy Approach}

\subsection{Intuitionistic Fuzzy K Means (IFKM)}

Intuitionistic fuzzy K-means clustering algorithm is based upon Intuitionistic fuzzy set theory. Fuzzy set generates only membership function $\mu(\mathrm{x}), \mathrm{x} \in \mathrm{X}$, whereas Intuitionistic fuzzy set (IFS) given by Atanassov considers both membership $\mu(\mathrm{x})$ and non-membership $v(\mathrm{x})$. An Intuitionistic fuzzy set $\mathrm{A}$ in $\mathrm{X}$, is written as the equation (12)

$$
A=\left\{x, \mu_{A}(x), v_{A}(x) \mid x \in X\right\}
$$

where $\mu_{A}(x) \rightarrow[0,1], v_{A}(x) \rightarrow[0,1]$ are the membership and non-membership degrees of an element in the set $\mathrm{A}$ with the condition as mentioned in the equation (13)

$$
0 \leq \mu(x)+v_{A}(x) \leq 1
$$

When $\mu \mu_{A}(x) \rightarrow[0.1], v_{A}(x) \rightarrow[0.1]$ for every $x$ in the set $\mathrm{A}$, then the set A becomes a fuzzy set. For all Intuitionistic fuzzy sets, Atanassov also indicated a hesitation degree, $\pi_{A}(x)$ which arises due to lack of knowledge in defining the membership degree of each element, $\mathrm{x}$ in the set $\mathrm{A}$ and is given by the equation (14)

$$
\pi_{A}(x)=1-\mu_{A}(x)-v_{A}(x) ; 0 \leq \pi_{A}(x) \leq 1
$$

Due to hesitation degree, the membership values lie in the interval as mentioned in the equation (15)

$$
\left[\mu_{A}(x), \mu_{A}(x)+\pi_{A}(x)\right]
$$

IFKM minimizes the objective function as in equation (16)

$$
\begin{gathered}
I_{I F K M}=\sum_{r=1}^{k} J_{r} \\
\sum_{r=1}^{k} \sum_{i=1}^{n} \mu_{r i}^{* m} d\left(x_{i}, c_{r}\right)^{2}+\sum_{r=1}^{k} \pi_{r}^{*} e^{1-\pi_{r}^{*}}
\end{gathered}
$$

where,

$$
\mu_{r i}^{*}=\mu_{r i}+\pi_{r i}
$$

where $\mu_{r i}^{*}$ denotes the Intuitionistic fuzzy membership and $\mu_{r i}$ denotes the conventional fuzzy membership of the $\mathrm{i}^{\text {th }}$ feature in $\mathrm{r}^{\text {th }}$ cluster defined as follows in the equation (17)

$$
\begin{array}{r}
U_{1} r i=\frac{1 /\left(\Sigma_{1}(l-1)^{\dagger} k \overline{\underline{3}}\left(\left(d\left(x_{1} i, c_{1} r\right)\right)\right) /\right.}{\left.\left(d\left(x_{1} i, c_{1} l\right)\right)^{\dagger}(2 /((m-1)))\right)}
\end{array}
$$

In this paper we adopted the distance measure proposed by D. M. Tsai and C. C. Lin. they incorporated a new distance measure into the conventional FCM as equation (19)

$$
d\left(x_{i}, c_{r}\right)=\frac{\left\|x_{i}-c_{r}\right\|^{2}}{\sigma_{r}}
$$

and $\sigma$ is the weighted mean distance of cluster $r$ and is given by equation (20)

$$
\sigma_{r}=\left\{\frac{\sum_{i=\mathbf{1}}^{n} \mu_{r i}^{* m} \cdot\left\|x_{i}-c_{r}\right\|^{2}}{\sum_{i=\mathbf{1}}^{n} \mu_{r i}^{* m}}\right\}
$$

$\pi_{r i}$ is hesitation degree, which is defined as follows in the equation (21)

$$
\pi_{r i}=1-\mu_{r i}-\left(1-\mu_{r i}^{\alpha}\right)^{1 / \alpha}, \quad \alpha>0
$$

and is calculated from Yager's Intuitionistic fuzzy complement, the Intuitionistic fuzzy set becomes(22)

$$
\begin{gathered}
A^{I F S}=\left\{x, \mu_{A}(x),\left(1-\mu_{A}(x)^{\alpha}\right)^{1 / \alpha} \mid x \in X\right\} \\
\pi_{r}^{*}=\frac{1}{N} \sum_{i=1}^{n} \pi_{r i}
\end{gathered}
$$

Step1: Initializes the $\mathrm{k}$ Intuitionistic fuzzy cluster centers randomly

Step2: while the stop condition is not satisfied do the following:

a) Compute the Intuitionistic Fuzzy membership and indeterministic value of each data point according to the equations (17) and (23)

b) Update Intuitionistic fuzzy cluster centers according to equation (20)

For each features with different membership and indeterministic values assigned to $\mathrm{k}$ different clusters. Because in Intuitionistic fuzzy each feature may not belong to just one cluster and it is much better to, consider the correlation of each feature to features in entire clusters.

Hence, during the selection of the best features, more accurate relations between genes are available. The main point here is that in selecting each feature, it is considered among the entire clusters not just one. Hence, in this case a feature, which is not, correlated enough with members of one cluster but its correlation among entire clusters is high, gains more chance to be selected in comparison with crisp attribute clustering techniques. 
This method uses the interdependence redundancy measure as the similarity measure. To cluster features, we used the Intuitionistic k-modes algorithm in which mode of each cluster is defined as one of its features which has the largest multiple interdependence redundancy measure among other features in that cluster.

A modified version of (6), where distance functional is defined as the inverse of interdependency measure

$$
\begin{aligned}
\mu_{r i}^{*}= & \frac{1}{\sum_{i=1}^{k}\left[\frac{R\left(A_{i}, n_{l}\right)}{R\left(A_{i}, n_{r}\right)}\right]^{2 / m-1}}+\left[1-\frac{1}{\sum_{i=1}^{k}\left[\frac{R\left(A_{i}, n_{l}\right)}{R\left(A_{i}, n_{r}\right)}\right]^{2 / m-1}}\right. \\
& \left.-\left[1-\left[\frac{1}{\sum_{i=1}^{k}\left[\frac{R\left(A_{i}, n_{l}\right)}{R\left(A_{i}, n_{r}\right)}\right]^{2 / m-1}}\right]^{\alpha}\right]^{1 / \alpha}\right]
\end{aligned}
$$

where, $l i \mathrm{~s}$ number of clusters. $\mu_{r i}^{*}$ is Intuitionistic fuzzy membership degree of $i^{\text {th }}$ feature in $r^{\text {th }}$ cluster and $\mathrm{m}$ is a weighting exponent. Afterwards, to satisfy the condition (4) $\mu_{r i}^{*}$ is normalized by equation (25)

$$
\mu_{r i}^{*}=\frac{\mu_{r i}^{* n e w}}{\sum_{l=1}^{k} \mu_{l i}^{* o l d}}
$$

In this approach mode of a cluster is updated to the feature with the highest fuzzy multiple interdependence redundancy in that cluster. Fuzzy multiple interdependence redundancy of each feature should be calculated regardless of its own membership degree in associated cluster. Considering this Intuitionistic fuzzy membership degree, mode of each cluster will never change, since it has high membership degree in that cluster

\subsection{Novel Genetic Algorithm Based Intuitionistic Fuzzification Techniques in Selection of Features from Clusters}

After Clustering of features, the best one should be selected from each cluster. In this paper genetic algorithm technique is proposed in order to consider Intuitionistic fuzzy memberships of features in clusters during selection. At first each feature is consider to belong to a cluster which has highest membership degree. Members of $\mathrm{r}^{\text {th }}$ cluster are represented by member $r$. Rank of each feature in its cluster is calculated and the one with the highest ranks are selected as the candidate. For calculating the rank of each feature in /Intuitionistic fuzzy approach technique assigns a positive weight to the inter-dependency of features which are belonged to membership to cluster $r$. It also assigns a negative weight as a penalty to the interdependency of features as non membership to cluster $r$.

The assigned weight is equal to the membership degree of the feature in its cluster.

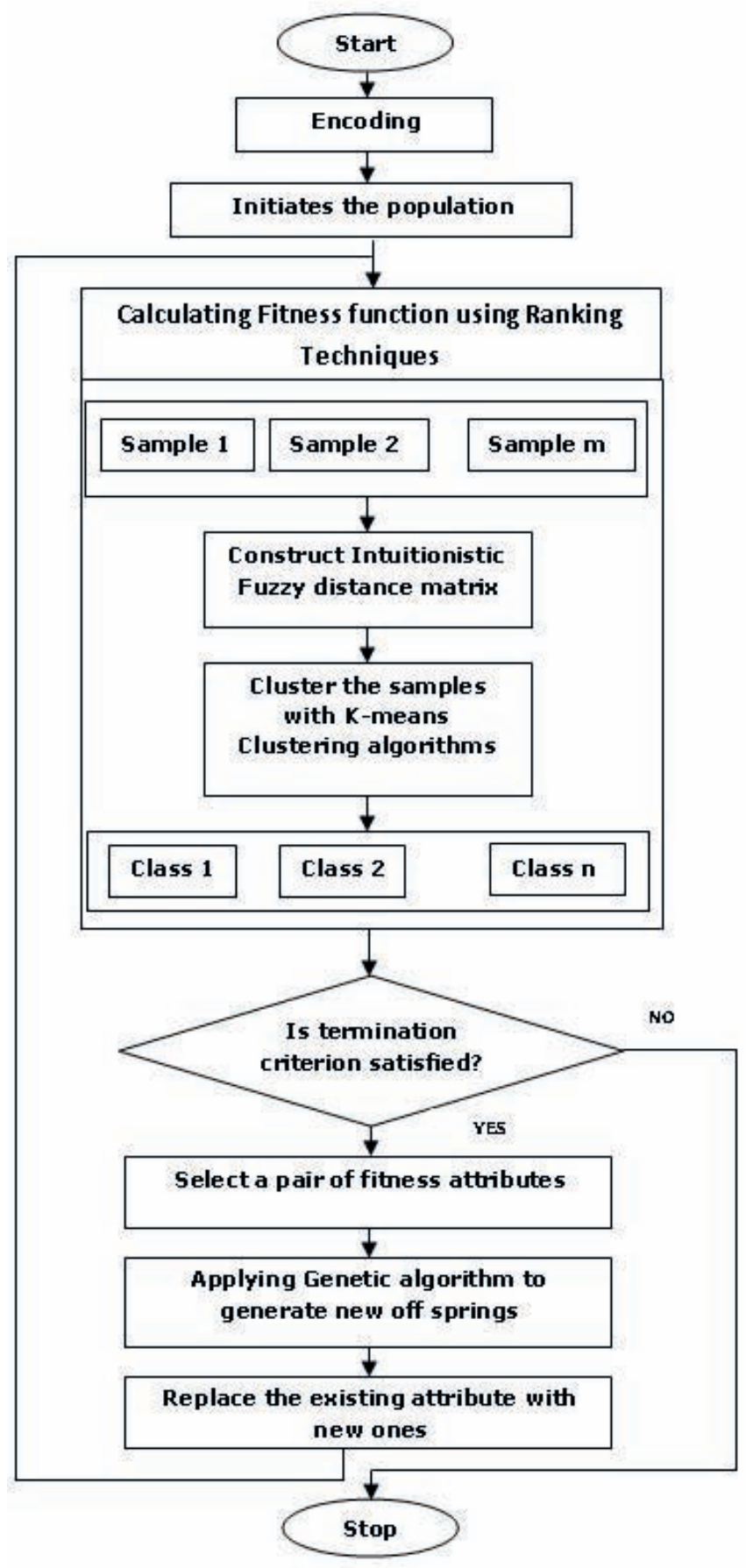

Figure 1. Flow structure of Genetic Intuitionistic Fuzzy Approach. 
Therefore, the selected feature would be more interdependent with its co-cluster features and less interdependent with other clusters' members. Calculating the rank of each feature in this method is performed according to (26).

$$
\begin{aligned}
\operatorname{rank}_{r}\left(A_{i}\right)= & \mu_{r i}^{* m}\left[\sum_{j \in m e m b e r s_{r}, j \neq i} \mu_{r j}^{* m} R\left(A_{i}: A_{j}\right)\right] \\
& -\mu_{r i}^{* m}\left[\sum_{j \in \text { members } s_{r . j} \neq i} \mu_{r j}^{* m} R\left(A_{i}: A_{j}\right)\right]
\end{aligned}
$$

It is considered as the fitness function of the genetic algorithm. The features with highest fitness rank are selected as the best candidates to perform the crossover operation. The genetic algorithm uses in addition the insertion operator at particular iterations to overcome the problem of local optima. So it adapts the concept of genetic algorithm restarting in which it deletes some of the instances from current population and adds the same amount of instances from the initial population which is left during the initial stage of random population generation.

Evaluation of feature ranking technique is considered as the fitness function. The candidates with the highest ranking is considered as the holding the highest fitness value and they are taken into the consideration for further processing in genetic algorithm restarting. Figure-1 shows the flow structure of genetic Intuitionistic fuzzy approach for selecting attributes.

\section{Results}

The results reports of the proposed method, performed with the classification produced with the results on these Sensor, Iris and Breast cancer Data sets. The data sets are split into $70 \%$ of training data and $30 \%$ of testing data. Like what we did in this data set, we used J4.8 Decision tree method to build classifiers using selected genes as the training set. The Classifiers thus built were on the samples in the data set. The Classification accuracy can be used for assessing the feature selection methods. J4.8 decision tree is applied for assessing the classification based on Comparison of Classification results with number of Clusters and Whole features for K-Means, Spanning Tree Attribute Clustering Algorithm (ST-ACA), Attribute Clustering Algorithm (ACA) in comparison with Fuzzy Attribute Clustering Algorithm (FACA) and Intuitionistic Fuzzy based Attribute clustering Algorithm are demonstrated below and results are represented in the Table 1 and Performance are presents in Graphical representation in below Figure 2.
Table 1. Comparison of classification results with number of clusters and whole features are presented

\begin{tabular}{lccccc}
\hline Dataset & Sensor & Iris & $\begin{array}{c}\text { Breast } \\
\text { Cancer }\end{array}$ & Diabetes & $\begin{array}{c}\text { Heart } \\
\text { disease }\end{array}$ \\
\hline $\begin{array}{l}\text { Number of } \\
\text { clusters }\end{array}$ & 50 & 20 & 30 & 15 & 25 \\
K-Means & 79.76 & 89.45 & 92.68 & 82.85 & 76.94 \\
ACA & 80.23 & 91.21 & 93.09 & 84.39 & 78.57 \\
ST-ACA & 82.67 & 90.89 & 92.69 & 86.78 & 82.47 \\
FACA & 86.71 & 92.80 & 94.94 & 92.26 & 94.18 \\
$\begin{array}{l}\text { Genetic } \\
\text { Intuitionistic }\end{array}$ & 90.95 & 96.07 & 96.38 & 95.59 & 96.74 \\
fuzzy approach & & & & & \\
\hline
\end{tabular}

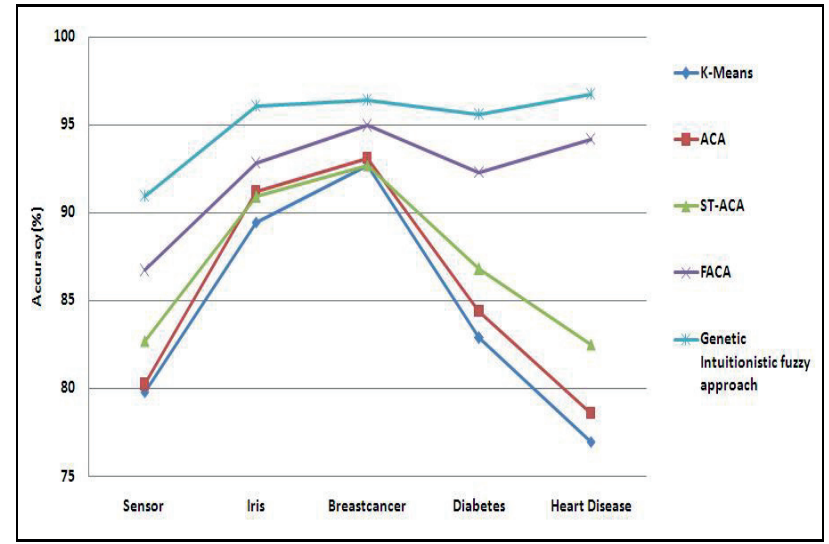

Figure 2. Comparison of Classification results based on different data sets with K-means, Spanning Tree ACA, ACA, FACA and Genetic Intuitionistic Fuzzy Approaches.

The proposed Method seems to more consistent than FACA, ACA, K-means and Spanning tree based Attribute based Clustering. Here, J4.8 decision tree is used to classify the Sensor, Iris, Breast Cancer, Diabetes and Heart Disease data set depending upon the selected features. The results of the classification accuracy can be encountered as an interdependency measure of accessing the feature selection method. Hence, proposed method, ACA, Fuzzy ACA, K-means and Spanning tree based Attribute based Clustering methods compare with the accuracy rate as depicted in Figure-2. Each feature belongs to the cluster with the maximum membership degree and it is assessed to generalized classification system. According to the obtain results, The Stability of Clustering in High or Low cluster numbers cannot be distinguishable. Therefore, only in these cases FACA give better results than k-means, Spanning Tree ACA, ACA. In all other cases proposed Method gains much better results than comparable within FACA. Based on the 
Experiments Results, it appears that pertinently selecting features improves considerably in classification results.

From Table 2 to 5 presents the removing unrelated clusters from the number of selected features in the different data set and compared proposed approach results with different inference system results.

Table 2. Comparison of methods according to the classification and number of selected features for sensor dataset (number of clusters $=25$ )

\begin{tabular}{lcc}
\hline Techniques & $\begin{array}{c}\text { Classification } \\
\text { Accuracy }\end{array}$ & $\begin{array}{c}\text { No. of Selected } \\
\text { Features }\end{array}$ \\
\hline K-Means & 73.02 & 25 \\
ST-ACA & 75.76 & 20 \\
ACA & 76.54 & 15 \\
FACA & 80.92 & 12 \\
Genetic Intuitionistic & 89.17 & 10 \\
fuzzy approach & & \\
\hline
\end{tabular}

As we can see, the novel approach i.e. proposed method removes 15 unrelated Clusters.

Table 3. Comparison of methods according to the classification and number of selected features for iris dataset (number of clusters $=15$ )

\begin{tabular}{lcc}
\hline Techniques & $\begin{array}{c}\text { Classification } \\
\text { Accuracy }\end{array}$ & $\begin{array}{c}\text { No. of Selected } \\
\text { Features }\end{array}$ \\
\hline K-Means & 74.09 & 14 \\
ST-ACA & 75.98 & 12 \\
ACA & 78.25 & 10 \\
FACA & 82.79 & 9 \\
Genetic Intuitionistic & 92.65 & 6 \\
fuzzy approach & & \\
\hline
\end{tabular}

As we can see, the novel approach i.e. proposed method removes 9 unrelated Clusters.

Table 4. Comparison of methods according to the classification and number of selected features for diabetes dataset (number of clusters $=30$ )

\begin{tabular}{lcc}
\hline Techniques & $\begin{array}{c}\text { Classification } \\
\text { Accuracy }\end{array}$ & $\begin{array}{c}\text { No. of Selected } \\
\text { Features }\end{array}$ \\
\hline K-Means & 76.54 & 30 \\
ST-ACA & 78.96 & 28 \\
ACA & 80.76 & 28 \\
FACA & 87.54 & 23 \\
Genetic Intuitionistic & 93.77 & 20 \\
fuzzy approach & & \\
\hline
\end{tabular}

As we can see, the novel approach i.e. proposed method removes 10 unrelated Clusters.

Table 5. Comparison of methods according to the classification and number of selected features for diabetes dataset (number of clusters $=20$ )

\begin{tabular}{lll}
\hline Techniques & $\begin{array}{l}\text { Classification } \\
\text { Accuracy }\end{array}$ & $\begin{array}{l}\text { No. of Selected } \\
\text { Features }\end{array}$ \\
\hline K-Means & 78.43 & 18 \\
ST-ACA & 80.92 & 16 \\
ACA & 83.76 & 15 \\
FACA & 88.74 & 12 \\
Genetic Intuitionistic & 94.06 & 11 \\
fuzzy approach & & \\
\hline
\end{tabular}

As we can see, the novel approach i.e. proposed method removes 9 unrelated Clusters.

Table 6. Comparison of methods according to the classification and number of selected features for heart disease dataset (number of Clusters $=60$ )

\begin{tabular}{lll}
\hline Techniques & $\begin{array}{l}\text { Classification } \\
\text { Accuracy }\end{array}$ & $\begin{array}{l}\text { No. of Selected } \\
\text { Features }\end{array}$ \\
\hline K-Means & 80.24 & 60 \\
ST-ACA & 81.73 & 54 \\
ACA & 84.59 & 52 \\
FACA & 86.18 & 49 \\
$\begin{array}{l}\text { Genetic Intuitionistic } \\
\text { fuzzy approach }\end{array}$ & 94.92 & 45 \\
\hline
\end{tabular}

As we can see, the novel approach i.e. proposed method removes 15 unrelated Clusters.

Table 7. Accuracy results of different datasets based on selecting features

\begin{tabular}{lccccc}
\hline \multirow{1}{*}{ Dataset } & \multicolumn{5}{c}{ Accuracy (\%) } \\
\cline { 2 - 6 } Techniques & Sensor & Iris & $\begin{array}{c}\text { Breast } \\
\text { Cancer }\end{array}$ & Diabetes & $\begin{array}{c}\text { Heart } \\
\text { disease }\end{array}$ \\
\hline K-Means & 73.72 & 70.36 & 75.39 & 72.09 & 76.93 \\
ACA & 74.58 & 73.63 & 78.94 & 75.63 & 77.95 \\
ST-ACA & 76.54 & 78.25 & 80.76 & 79.57 & 80.06 \\
FACA & 80.92 & 82.79 & 87.54 & 81.70 & 88.96 \\
Genetic & 89.17 & 92.65 & 93.77 & 93.73 & 92.82 \\
Intuitionistic & & & & & \\
fuzzy approach & & & & & \\
\hline
\end{tabular}

As we can see, the novel approach i.e. proposed method removes 9 unrelated Clusters. 
As we can see, the novel approach i.e. proposed method removes 15 unrelated Clusters.

The accuracy results can be used for assessing classification based on selected features for K-Means, Spanning Tree ACA, Attribute Clustering Algorithm (ACA) in comparison with Fuzzy Attribute Clustering Algorithm (FACA) and Intuitionistic Fuzzy based Attribute clustering Algorithm are demonstrated below and results are represented in the Table 7 and Performance are presents in Graphical representation in below Figure 3.

The Figure 4 to 8 shows the performance of the proposed method using the five different benchmark datasets with no.of clusters with the varying fitness value. The fitness function $f(n)$ is tested in a sequence of experiments on the five different uci machine learning datasets shown in Figure 4 to 8 to represent the robustness of $\mathrm{f}(\mathrm{n})$. In the

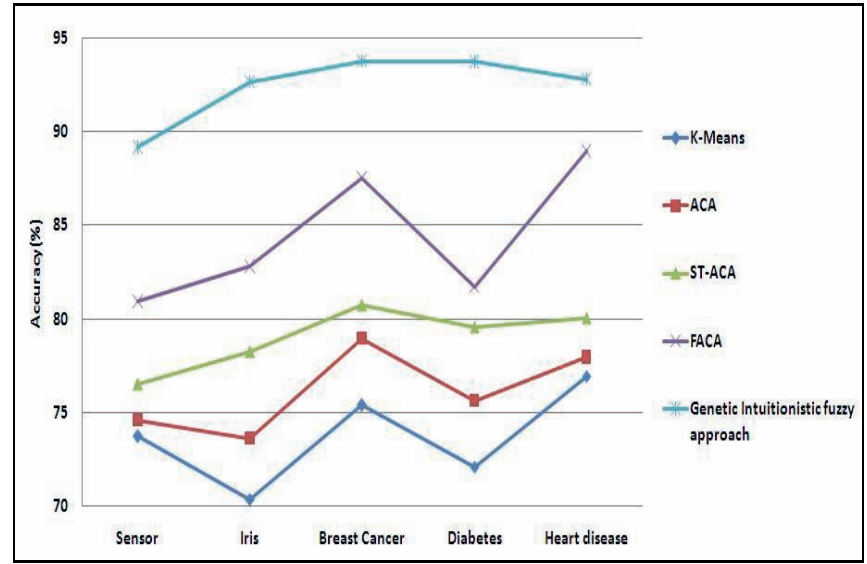

Figure 3. Performance chart based on different datasets with selecting features.

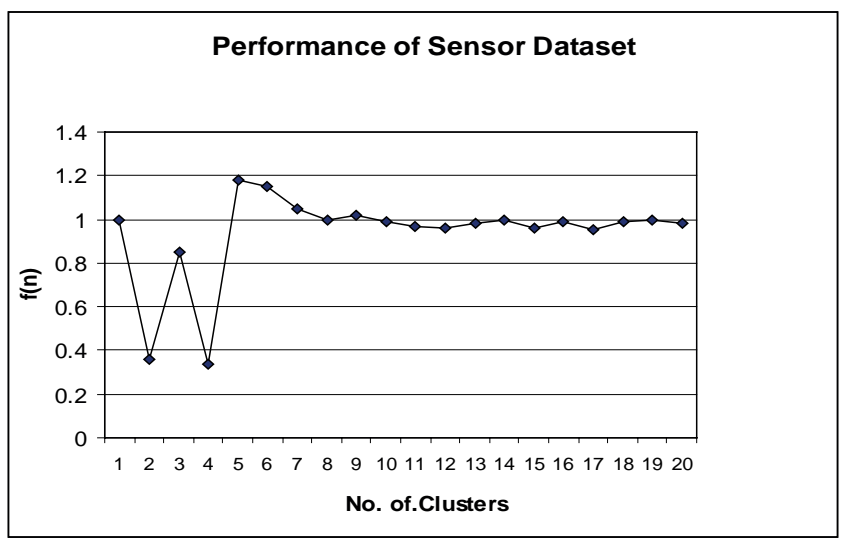

Figure 4. Performance of Sensor.

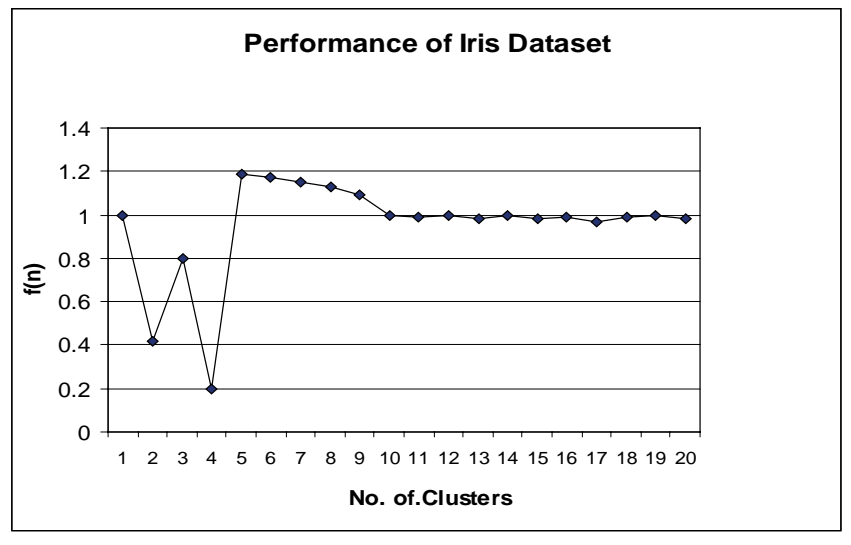

Figure 5. Performance of Iris.

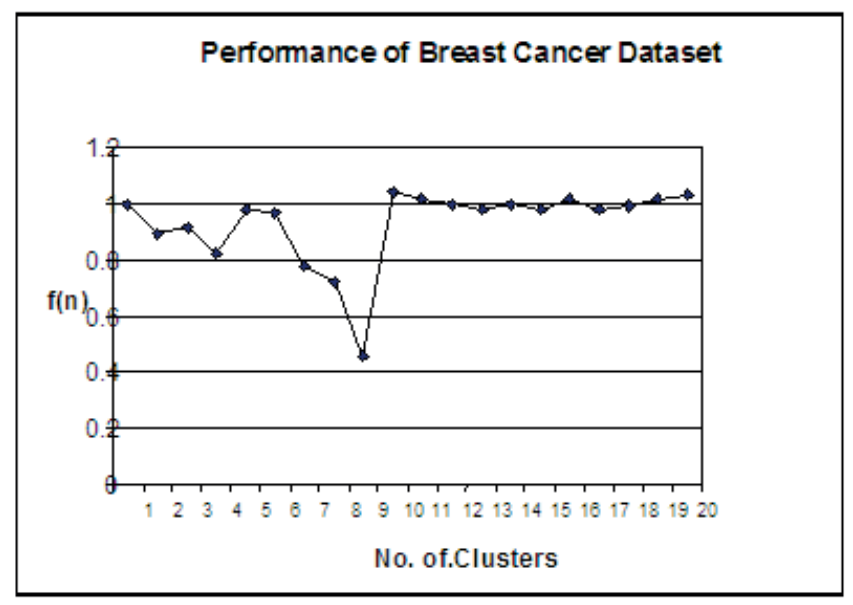

Figure 6. Performance of Breast Cancer.

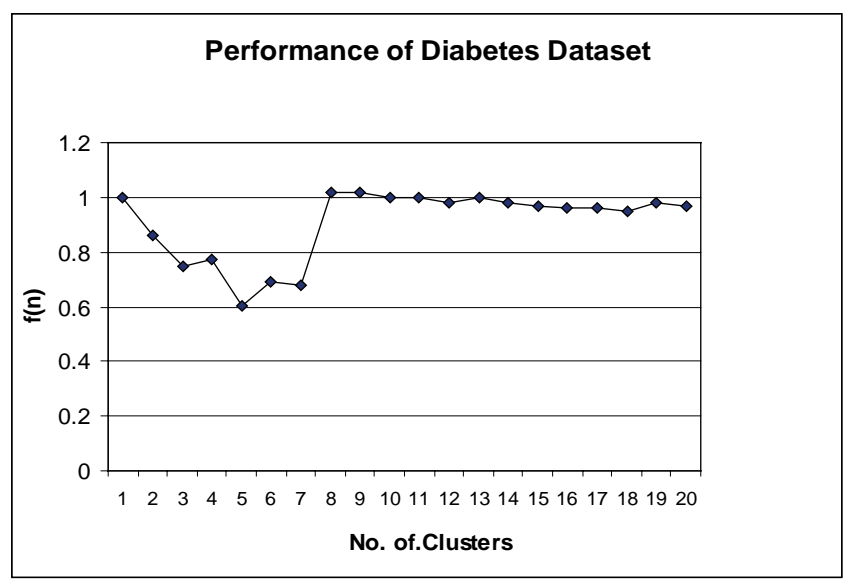

Figure 7. Performance of Diabetes.

Figure 4 the $\mathrm{f}(\mathrm{n})$ reaches minimum values at $\mathrm{n} 2$ and 4 respectively. In such cases, users could select the most appropriate value of $\mathrm{n}$ based on their specific requirements. 


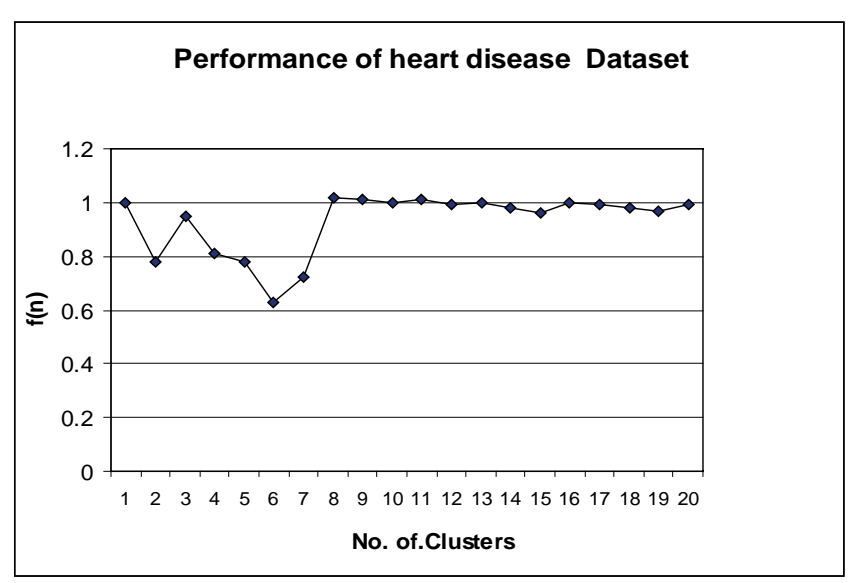

Figure 8. Performance of Heart disease.

For instance if a threshold of 0.85 is selected for $\mathrm{f}(\mathrm{n})$ the numbers of clusters recommended for each of these data sets lies around the range of 0.85 . Example in the heart disease dataset the number of recommended clusters is 2,3 and 4 . The fitness function converges in most cases to 1 when $\mathrm{K}$ increases above 9.

\section{Conclusion}

In this paper, an Intuitionistic fuzzy based clustering and selection of features is proposed based on genetic algorithm for removing redundant clusters and identifying the best potential features for further processing. This method not only improves classification accuracy, but also simplifies learning task by selecting less number of features to a great extent. Different novel techniques for selecting best feature from each cluster by extending the degree of membership to in deterministic value of instances. In future, based on this proposed work we construct a model for classification.

\section{Acknowledgement}

We thank Karpagam University for the motivation and encouragement for giving me the opportunity to do this research work as successful one.

\section{References}

1. Masud M M, Chen $\mathrm{O}$ et al. (2012). Classification and adaptive novel class detection of feature evolving data streams, IEEE Transactions on Knowledge and Data Engineering, vol $\mathrm{PP}(99)$.
2. Wu X, Yu K et al. (2012). Online feature selection with streaming features, IEEE Transactions on Pattern Analysis and Machine Intelligence, vol 35(99), 1178-1192.

3. Dai B, Huang J et al. (2006). Adaptive clustering for multiple evolving streams, IEEE Transactions on Knowledge and Data Engineering, vol 18(9), 1166-1180.

4. Song O, Ni J et al. (2013). A fast clustering-based feature subset selection algorithm for high-dimensional data, IEEE Transactions on Knowledge and Data Engineering, vol 25 (1), 1-14.

5. Chouaib H, Terrades $\mathrm{O}$ R et al. (2008). Feature selection combining genetic algorithm and Adaboost classifiers, 19th International Conference on Pattern Recognition, 2008. ICPR 2008, 1-4.

6. Wasikowski M, and Chen X (2010). Combating the small sample class imbalance problem using feature selection, IEEE Transactions on Knowledge and Data Engineering, vol 22(10), 1388-1400.

7. Guyon I, and Elisseeff A (2003). An introduction to variable and feature selection, Journal of Machine Learning Research, vol 3, 1157-1182.

8. Dash M, and Liu H (1997). Feature selection for classification, Intelligent Data Analysis, vol 1(1-4), 131-156.

9. Jain A K, and Zongker D E (1997). Feature selection: evaluation, application, and small sample performance, IEEE Transactions on Pattern Analysis and Machine Intelligence, vol 19(2), 153-158.

10. Koller D, and Sahami M (1996). Toward optimal feature selection, Proceedings 13th International Conference Machine Learning, 284-292.

11. Yu L, and Liu H (2004). Efficient feature selection via analysis of relevance and redundancy, Journal of Machine Learning Research, vol 5, 1205-1224.

12. Dash M, and Liu H (1997). Feature selection for classification, Intelligent Data Analysis, Elsevier Science B.V., vol. 1(3), 131-156.

13. Zhu Z, Ong Y et al. (2007). Wrapper filter feature selection algorithm using a memetic framework, IEEE Transactions on Systems, Man and Cybernetics, vol.37 (1), 70-76.

14. Au W H, Chan K C C et al. (2005). Attribute clustering for grouping, selection, and classification of gene expression Data, IEEE/ACM Transactions on Computational Biology and Bioinformatics, vol. 2(2), 83-101.

15. Wei H L, and Billings S A (2007). Feature subset selection and ranking for data dimensionality reduction. IEEE Transactions on Pattern Analysis and Machine Intelligence, vol. 29(1), 162-166.

16. Tan K C, Teoh E J et al. (2008). A hybrid evolutionary algorithm for attribute selection in data mining, Expert Systems with Applications, vol 36(4), 8616-8630.

17. Maji P (2012). Mutual information-based supervised attribute clustering for microarray sample classification, IEEE 
Transactions on Knowledge and Data Engineering, vol. 24(1), 127-140.

18. Javed K, Babri H A et al. (2012). Feature selection based on class-dependent densities for high-dimensional binary data, IEEE Transactions on Knowledge and Data Engineering, vol 24(3), 465-477.

19. Zhang S, Wong $\mathrm{H}$ et al. (2012). A new unsupervised feature ranking method for gene expression data based on consensus affinity, IEEE/ACM Transactions on Computational Biology and Bioinformatics, vol 9(4), 1257-1263.

20. Yang S H and $\mathrm{Hu} B$ G (2012). Discriminative feature selection by nonparametric bayes error minimization, IEEE Transactions on Knowledge and Data Engineering, vol 24(8), 1422-1434.

21. Esmaeili M, and Gabor F (2011). Feature selection as an improving step for decision tree construction, Proceedings of International Conference on Machine Learning and Computing, vol 3, 35-39.

22. Tsai D, and Lin C (2011). Fuzzy C-means based clustering for linearly and nonlinearly separable data, Pattern Recognition, vol 44 (8), 1750-1760.

23. Gonzalez A, and Pérez R (2001). Selection of relevant features in a fuzzy genetic learning algorithm, IEEE Transactions on Systems, Man and Cybernetics, vol 31(3), 417-425.

24. Huang C L, and Wang C (2006). A GA-based feature selection and parameters optimization for support vector machines, Expert Systems with Applications, vol 31(2), 231-240.

25. Tan F, Fu X (2008). A genetic algorithm-based method for feature subset selection, Soft Computing, vol 12 (2).111-120.

26. Hoi C H S, Wang J et al. (2012). Online feature selection for mining big data, BigMine'12, 93-100.

27. Chitsaz E, Taheri M et al. (2008). A Fuzzy approach to clustering and selecting features for classification of gene expression data, Proceedings of the World Congress on Engineering, vol II.
28. Chang C T, Lai J Z C et al. (2011). A Fuzzy K-means clustering algorithm using cluster center displacement, Journal of Information Science and Engineering, vol 27, 995-1009.

29. Atanassov K T (1986). Intuitionistic fuzzy sets, Fuzzy Sets and Systems, vol20, 87-96.

30. Al-Harbi S H, and Rayward-Smith V J (2006). Adapting $\mathrm{k}$-means for supervised clustering, Applied Intelligence, vol 24 (3), 219-226.

31. Chitsa Z E, Taheri M et al. (2009). An improved fuzzy feature clustering and selection based on Chi-Squared-Test, Proceeding of the International Multi Conference of Engineers and Computer Scientists.

32. Tsang E C C, Yeung D S et al. (2003). OFFSS: Optimal fuzzy-valued feature subset selection, IEEE Transactions on Fuzzy Systems, vol11 (2), 202-213.

33. Pizzileo B, Li K et al. (2012). Improved structure optimization for fuzzy-neural networks, IEEE Transactions on Fuzzy Systems, vol 20(6), 1076-1089.

34. Arima C, Hanai T et al. (2003). Gene expression analysis using fuzzy k-means clustering, Genome Informatics, vol 14(1), 334-335.

35. Deng Z, Chung F L et al. (2010). Robust relief-feature weighting, margin maximization and fuzzy optimization, IEEE Transactions on Fuzzy Systems, vol 18(4), 726-744.

36. Yager R R (2009). Some aspects of Intuitionistic Fuzzy sets, Fuzzy Optimization and Decision Making, vol 8(1), 67-90.

37. Hu O, Pan W et al. (2012). Feature selection for monotonic classification, IEEE Transactions on Fuzzy Systems, vol 20(1), 69-81.

38. Maji P (2011). Fuzzy rough supervised attribute clustering algorithm and classification of microarray data, IEEE Transactions on Systems, Man, and Cybernetics, vol 41(1), 222-233. 